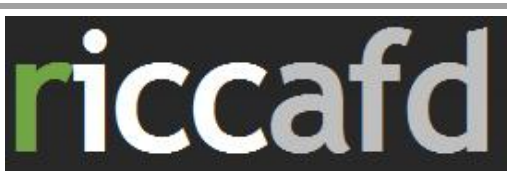

Revista Iberoamericana de Ciencias de la Actividad Física y el Deporte

\title{
INFLUENCIA DEL ESTIRAMIENTO EN EL CALENTAMIENTO PARA EL SALTO Y LA VELOCIDAD
}

\section{INFLUENCE OF STRETCHING ON WARM UP IN JUMP AND SPEED}

Gálvez Ruiz, P.

Doctor por la Universidad de Málaga. Ldo. en CC. de la Actividad Física y el Deporte. pab_galvez@hotmail.com

Tapia Flores, A.

Doctor por la Universidad de Málaga. Ldo. en CC. de la Actividad Física y el Deporte

Jurado Lavanant, $\mathbf{A}$.

Ldo. en CC. de la Actividad Física y el Deporte. Master Investigación en Actividad Física y Deporte

Código UNESCO: 5802.99

Clasificación Consejo de Europa: 5

Recibido el 5 de abril de 2013

Aceptado el 1 de junio de 2013

PALABRAS
CLAVE:
Calentamiento,
Estiramiento,
Fuerza,
Velocidad

Se han encontrado disminuciones en el rendimiento de los test de SJ y RJ, así como también en el test de velocidad, obteniéndose valores significativos $(p<0,05)$ tanto para la potencia y el tiempo de vuelo del test RJ, como también para el test de velocidad. En cuanto al test CMJ, se han obtenido beneficios aunque las diferencias no han sido significativas. Por tanto, la realización de estiramientos pasivos influye de forma negativa en el rendimiento de la fuerza explosiva y la velocidad en una muestra de futbolistas entrenados.

KEY WORDS:
Warm Up,
Stretching,
Strength,
Speed

\begin{abstract}
The purpose of the study is to determine the influence of passive stretchings included as part of warm up in explosive strength performance, in this case in several vertical jump tests: Squat Jump (SJ), Countermouvement Jump (CMJ) and Reactive Jump (RJ); and also in a 30 meters speed test with 5 meters start flying.

Sample was composed by 9 male Málaga Football Club soccer players $(18.22 \pm 0.44$ years) performed 2 warm up protocols in they free day in two consecutive weeks. The soccer players use the passive stretching in warm up and the kind of effort during the activity has a high component of explosive ballistic strength, movements executed by great intensity and short duration, for example shoot the ball, sprints, way changes, jumps, etc.

Were observed decreases in SJ, RJ and speed tests performance, with statistic significant differences $(p<0.05)$ in power and flight time of RJ test, also in speed test. As for the CMJ test, benefits have
\end{abstract} been obtained though the differences have not been significant. Therefore, passive stretching included in warm up had a negative influence in explosive strength and speed in trained soccer players group. 


\section{INTRODUCCIÓN}

La realización de un calentamiento justo antes de comenzar un ejercicio físico de cualquier intensidad es algo comúnmente aceptado y practicado por deportistas, ya que con ello, se evitan posibles lesiones y se garantiza una mejor realización en cada ejercicio ${ }^{1-4}$.

No obstante, el calentamiento consiste en una serie de ejercicios y actividades realizadas inmediatamente antes de la actividad principal, que ayudan al cuerpo a readaptarse desde un estado de descanso al ejercicio ${ }^{3} ; 0$ dicho de otra manera, son los ejercicios que disponen las funciones orgánicas, nerviosas, psicológicas, etc. del deportista para un rendimiento óptimo en la actividad a realizar.

Existe de hecho, una amplia literatura tanto de los efectos como de los beneficios que aporta el calentamiento en la posterior actividad física $^{5-11}$, ya sea a nivel fisiológico (sistema circulatorio, cardiovascular, respiratorio, muscular y/o nervioso) psicológico y/o educativo.

Los tipos de ejercicios que se incluyen en el calentamiento difieren en función de la actividad que se vaya a realizar o el tipo de objetivo que se pretenda conseguir (en el caso de una sesión o entrenamiento), ya que el nivel de solicitación, tanto de los diferentes grupos musculares como de los sistemas del organismo, será muy distinto. Esto hace que al prescribir un calentamiento no haya un acuerdo sobre las partes que deben componer el mismo, así como la duración, intensidades, tipos de ejercicios (generales o específicos) y su orden $3,5,10,12,13$.

Los estiramientos suelen ser una de las partes fundamentales de todo calentamiento, existiendo diversas investigaciones que avalan la importancia que tiene mantener una flexibilidad adecuada u óptima, reconociéndola además como un componente importante de la condición física de los deportistas ${ }^{11,14,15}$.

Centrándonos en nuestra investigación, diversos estudios han mostrado que la realización de estiramientos pasivos antes de la actividad física pueden disminuir el rendimiento en acciones de fuerza máxima y velocidad ${ }^{16-21}$, siendo los motivos un cambio en la tensión músculo - tendinosa o una disminución de la activación del músculo. De cualquier forma, la previa realización de ejercicios de estiramiento pasivo podría influir negativamente sobre el rendimiento en actividades que implican el ciclo acortamiento - estiramiento.

El estiramiento pasivo o estático describe el grado en que se puede mover una articulación de forma pasiva hasta el límite de su movimiento ${ }^{22}$, lo que implica el estiramiento pasivo lento de un músculo para inhibir la activación del reflejo de estiramiento, colocándolo en una posición de

Rev. Ib. CE. Act.Fis. Dep. 
extensión máxima del individuo y manteniéndolo así durante un lapso prolongado de tiempo ${ }^{1,22}$.

Al igual que ocurre con el calentamiento, algunos autores constatan la gran importancia que tiene la realización de ejercicios de estiramiento para la mejora del rendimiento y para la prevención de lesiones ${ }^{5,6,15,23}$. De hecho, haciendo referencia a las lesiones, diversos estudios indican que tener una adecuada flexibilidad muscular podría evitar una serie de lesiones por sobreestiramiento, sobre todo en futbolistas, tales como desgarros y distensiones ${ }^{6,24,25}$. Sin embargo, una revisión ${ }^{26}$ en la que sólo se estudiaron a personas jóvenes, concluye que el estiramiento antes y/o después del ejercicio no ejerce ningún efecto protector sobre el dolor muscular ni reduce el riesgo de lesión.

De cualquier forma, en la literatura no existe un acuerdo sobre cuánto tiempo deben durar los estiramientos como parte del calentamiento $^{2,12}$, ni tampoco el tiempo concreto que se debe mantener el ejercicio de estiramiento para conseguir un rendimiento posterior óptimo. En este sentido, diversos autores ${ }^{27,28}$ afirman que el ejercicio de estiramiento pasivo debe durar entre 20-30 segundos, llegando hasta los 60 segundos si fuera necesario. En otros estudios ${ }^{7,22,29}$, indican tiempos que oscilan entre los 3 y 120 segundos de elongación, aunque debemos considerar que la población utilizada para estos estudios no ha sido de futbolistas ${ }^{30}$.
Diversos estudios realizados con futbolistas ${ }^{31,32}$, sugieren que los jóvenes jugadores que siguen un programa de entrenamiento pliométrico y que compiten regularmente obtienen valores superiores en los test Squat Jump (SJ), Countermouvement Jump (CMJ) y Drop Jump (DJ). Por otro lado, también se han encontrado relaciones entre la fuerza explosiva y las aceleraciones de 10 metros con cambios de dirección de $90^{\circ}$, en futbolistas de entre 18 y 21 años ${ }^{33}$.

La posición de juego en el campo también influye en los datos obtenidos en los test SJ y CMJ, de forma que tanto porteros, como defensas centrales y delanteros muestran valores más altos en los test SJ y CMJ que el resto de posiciones $^{34-38}$.

En la línea de nuestro trabajo, se encontró ${ }^{17}$ que los estiramientos previos reducían el rendimiento en la altura del salto en SJ $(4,4 \%)$ y CMJ $(4,3 \%)$, conclusión igualmente obtenida en otro estudio $^{21}$, lo que determina que los estiramientos estáticos tienen una influencia negativa en el rendimiento del salto vertical.

Contrariamente, hay estudios $^{16}$ que concluyen que la realización de un calentamiento con estiramientos pasivos no es el que mejores resultados consigue en el posterior test de salto vertical (CMJ) con respecto a otros protocolos de calentamiento, así como también se obtienen $^{20}$ diferencias significativas en los niveles de rendimiento en una repetición máxima de los

Rev. Ib. Cᄃ. Act.Fis. Dep. 
músculos flexo-extensores de los miembros inferiores, después de 10 minutos de estiramientos estáticos de cuadriceps y gemelos.

En cuanto a la influencia de los estiramientos en el rendimiento de pruebas de velocidad, un estudio $^{19}$ en el que realizaron diferentes protocolos de estiramientos en jugadores de rugby de competición para comprobar los efectos que producían en una prueba de velocidad de $20 \mathrm{mts}$., encontraron que el grupo que realizaba estiramientos pasivos mostraba un aumento significativo del tiempo en la prueba $(p<0.05)$.

Por tanto, estos estudios indican que los estiramientos pasivos son negativos para las posteriores acciones explosivas, tales como una prueba de velocidad o un test de salto vertical. De esta manera, el propósito de nuestro estudio era contrastar los datos encontrados en la literatura, realizando en nuestro caso varios tipos de acciones explosivas de manera continuada (diferentes tests de salto vertical y una prueba de velocidad máxima), pero sin que ello produjera la aparición de fatiga en los participantes para comprobar si la realización de estiramientos pasivos beneficia, perjudica 0 no influye en el posterior rendimiento físico.

\section{MATERIAL Y MÉTODO}

Participantes.

La muestra se compone de 9 jugadores de fútbol de categoría juvenil, de género masculino, con edad media de 18.22 años (DT=.44). Los participantes, pertenecientes al Málaga Club de Fútbol, están entrenados y compiten de manera regular dentro de la liga nacional de su categoría.

Tabla 1. Estadísticos descriptivos de la muestra de estudio: edad, IMC,

\begin{tabular}{lcccc}
\multicolumn{1}{c}{ peso y talla } & & & & \\
\hline \multicolumn{1}{c}{ Variable } & $\mathrm{N}$ & Mínimo & Máximo & Media \pm D.S. \\
\hline Edad (años) & 9 & 18 & 19 & $18.22 \pm .44$ \\
IMC día 1 $(\mathrm{kg} / \mathrm{m} 2)$ & 9 & 20.10 & 30.30 & $24.10 \pm 3.28$ \\
IMC día 2 & 9 & 17.90 & 28.30 & $22.41 \pm 3.55$ \\
Peso día 1 $(\mathrm{kg})$. & 9 & 68.20 & 86.90 & $78.45 \pm 5.96$ \\
Peso día 2 $(\mathrm{kg})$. & 9 & 67.00 & 85.00 & $76.53 \pm 5.84$ \\
Talla (cms.) & 9 & 170 & 191 & $178.00 \pm 6.24$ \\
\hline
\end{tabular}

Diseño.

El estudio se ha llevado a cabo mediante un diseño cuasiexperimental intrasujeto. En este caso, cada uno de los tratamientos se administra al mismo grupo de participantes $^{39}$, manipulando la variable independiente "estiramiento pasivo", para conocer si existe Rev. Ib. CL. Act.Fis. Dep. influencia sobre las variables dependientes "SJ, CMJ, RJ y test de velocidad", las cuales se describen a continuación:
A. Test de Bosco ${ }^{40}$ : se han utilizado las pruebas $S J, C M J$ y $R J$.
B. Test de velocidad máxima de carrera $^{41}$. Para valorar la 
velocidad de carrera máxima se emplean distancias no superiores a 60 metros, en la que sólo se controlan los 30-40 últimos metros y cuya salida es lanzada. En nuestro estudio, se utilizaron 5 metros para la fase de aceleración y se midió el tiempo que tardaban en recorrer los siguientes 30 metros utilizando para ello células fotoeléctricas dispuestas a los 0 y 30 metros respectivamente.

Se establecieron dos protocolos de calentamiento:

- Protocolo día 1 (P-1): activación vegetativa (5 minutos de carrera continua) y ejercicios de movilidad específica: $3 \times 10 \mathrm{mts}$. skipping, $3 \times 10 \mathrm{mts}$. talones al glúteo y $3 \times 20 \mathrm{mts}$. en segundo paso de triple. Una vez concluida cada serie de segundo paso de triple, se realizaba un sprint de $10 \mathrm{mts}$. La recuperación para cada una de las repeticiones consistía en volver andando sobre la distancia recorrida.

- Protocolo día 2 (P-2): incluía el mismo contenido que el protocolo P-1, añadiendo seis ejercicios de estiramiento pasivo durante 30 segundos cada uno al final del protocolo (gemelos contra la pared, isquiotibiales con flexión de $90^{\circ}$ de la cadera, cuádriceps con flexión de rodilla, aductores con espagat, psoas con lunch y glúteos con flexión de cadera y rodilla).

Desde el punto de vista fisiológico, se han tenido en cuenta los sistemas energéticos para establecer el orden de ejecución de los test. Tanto para la realización de las pruebas del Test de Bosco como del test de velocidad, los sistemas energéticos predominantes son el anaeróbico aláctico $\mathrm{y}$, en menor medida, el anaeróbico láctico ${ }^{41,42}$. EI sistema energético anaeróbico aláctico, que interviene durante los primeros diez segundos de ejercicio, cuenta con las reservas de adenosintrifosfato (ATP) y creatinfosfato (CP) almacenadas en las células musculares como fuente de energía rápida para actividades cortas y explosivas ${ }^{42}$, pero al proporcionar una cantidad de energía muy limitada, a los pocos segundos le sigue un descenso marcado de la potencia ${ }^{43}$. Es por ello que los test de saltos se realizaron en primer lugar, ya que suponen un esfuerzo de muy corta duración (SJ: <1seg., CMJ: <1seg. y RJ: $<5$ seg.), garantizando una óptima recuperación de los participantes para realizar el test de velocidad máxima.

Procedimiento.

Los participantes en la presente investigación fueron informados tanto verbalmente como por escrito del contenido del mismo, obteniendo su consentimiento para la realización de las pruebas específicas en base a las normas establecidas.

Se recopiló información personal, antropométrica y también del número de horas de entrenamiento semanal, de forma previa a la realización del protocolo establecido para las distintas pruebas. Así, la información recogida incluyó nombre y apellidos 
(posteriormente codificados en la base de datos), fecha de nacimiento, peso, talla, índice de masa corporal (IMC), posición de juego, número de horas de entrenamiento semanales $y$, por último, minuto de comienzo del calentamiento.

$\begin{array}{clll}\text { Los } & \text { protocolos } & \text { de } \\ \text { calentamiento } & (\mathrm{P}-1 \text { y } & \mathrm{P}-2) & \text { se }\end{array}$
llevaron a cabo de manera individual y entre el comienzo de cada participante se dispuso un intervalo de cinco minutos. Tras el respectivo calentamiento, se realizaron los diferentes test estipulados (SJ, CMJ, RJ y test de velocidad máxima de carrera), con el objetivo de conseguir los mejores resultados posibles.

Tanto la organización de los participantes, como el orden de ejecución de las pruebas fue el mismo en los dos protocolos, que se realizaron con un intervalo de tiempo de una semana completa. El día elegido para el desarrollo de cada protocolo fue la sesión de descanso. Todas las pruebas se llevaron a cabo en la pista de atletismo exterior del complejo deportivo de la Universidad de Málaga.

Instrumentos.

Se empleó una báscula Tefal de precisión (130kg./100gr.) y una cinta métrica calibrada de fibra de vidrio (marca Cóndor) para la valoración antropométrica. El Test de Bosco se desarrollo con la plataforma de infrarrojos Ergo Jump Plus Bosco System ${ }^{\circledR}$. Por último, la prueba de velocidad se evaluó mediante un sistema telemétrico de cronometraje (marca Byomedic), compuesto por dos pares de células fotoeléctricas.

Análisis estadístico.

Para el tratamiento de los datos se utilizó el software SPSS 15.0 para Windows. La normalidad de la muestra se comprobó mediante la prueba KolmogorovSmirnov. Además se realizó un análisis descriptivo de medias y desviaciones estándar de todas las variables del estudio. También se calculó la prueba $t$-Student para muestras relacionadas analizando las mismas variables en los dos protocolos.

\section{RESULTADOS}

Los resultados de la prueba Kolmogorov-Smirnov (K-S) mostró homogeneidad $(p<0.05)$ en todas las variables de estudio.

En la tabla 2 se muestran los datos descriptivos de las distintas pruebas de medida que constituyen la variable dependiente. En ella podemos observar que no se obtienen mejoras en los valores medios obtenidos correspondientes al P-2, salvo en el test CMJ, donde los resultados se ven beneficiados. Al realizar la prueba $t$ para muestras relacionadas, obtenemos valores significativos $(p<0.05)$ en el test de velocidad, así como también en la potencia de los saltos 2, 3 y 4 , y en el tiempo de contacto de los saltos 3 y 4 del test de RJ.

Rev. Ib. CC. Act.Fis. Dep. 
Tabla 2. Estadísticos descriptivos de las pruebas de la variable dependiente

\begin{tabular}{lccc}
\hline \multicolumn{1}{c}{ Variable } & \multicolumn{3}{c}{ Media \pm S.D. (día 1 vs. día 2) } \\
\hline VELOCIDAD & $3.69 \pm .13$ & vs & $3.88 \pm .17^{*}$ \\
SJ ALTURA & $.406 \pm .06$ & vs & $.397 \pm .05$ \\
CMJ ALTURA & $.408 \pm .05$ & vs & $.42 \pm .05$ \\
RJ VUELO salto 1 & $.539 \pm .02$ vs & $.554 \pm .03$ \\
RJ POTENCIA salto 1 & $37.615 \pm 11.22$ vs & $30.392 \pm 4.37$ \\
RJ VUELO salto 2 & $.557 \pm .03$ vs & $.544 \pm .04$ \\
RJ POTENCIA salto 2 & $39.626 \pm 12.38$ vs & $28.83 \pm 3.77^{*}$ \\
RJ VUELO salto 3 & $.56 \pm .01$ vs & $.547 \pm .03^{*}$ \\
RJ POTENCIA salto 3 & $37.266 \pm 8.87$ vs & $28.73 \pm 4.60^{*}$ \\
RJ VUELO salto 4 & $.553 \pm .02$ vs & $.553 \pm .03^{*}$ \\
RJ POTENCIA salto 4 & $36.427 \pm 9.41$ vs & $29.595 \pm 5.00$ * \\
\hline
\end{tabular}

$\left(^{*}\right)$ diferencias significativas $(p<0,05)$.

\section{DISCUSIÓN}

Los datos obtenidos en nuestro estudio permiten observar que la aplicación de ejercicios de estiramiento pasivo dentro del calentamiento no proporciona beneficios en el rendimiento de pruebas explosivas. En este sentido, los resultados encontrados coinciden con los expuestos en diversos estudios ${ }^{15,17,18,19,20}$, los cuales concluían que la realización de estiramientos pasivos antes de la actividad física provocaba una disminución en acciones de fuerza máxima y velocidad.

Como se ha podido observar en la tabla 4, donde se exponen los datos descriptivos, al aplicar estiramientos pasivos se obtienen beneficios en el test de CMJ tanto en el tiempo de vuelo (1.5\%) como en la altura del salto (2.5\%), contradiciendo así los resultados obtenidos en otras investigaciones 15,16. En éste último estudio ${ }^{16}$, encontraron una reducción del rendimiento en la altura del salto en SJ $(4.4 \%)$ y CMJ (4.3\%). No obstante, a pesar de la mejora obtenida en nuestro estudio en el test de CMJ, ésta no presenta valores significativos $(p<0.05)$.

Para las variables SJ y RJ, se produce una reducción del rendimiento al aplicar el protocolo con estiramientos pasivos. En el caso del test de SJ, dicha reducción no presenta valores significativos. Sin embargo, para el test RJ, comprobamos que existen diferencias significativas $(p<0.05)$ en la potencia de los saltos 2, 3 y 4, y en el tiempo de contacto 3 y 4 . No hemos encontrado en la literatura estudios que evalúen la influencia de los estiramientos pasivos en el rendimiento del test de $\mathrm{RJ}$, por lo que consideramos que son necesarias más investigaciones en este sentido.

En el caso del test de velocidad, se produce un aumento significativo $(p<0.05)$ del tiempo de ejecución en la prueba, lo que coincidiendo así con otro estudio ${ }^{18}$, que aplicaron a jugadores de rugby de competición diferentes protocolos de estiramientos $y$ realizaron la misma prueba de velocidad que nuestro estudio, encontrando un aumento

Rev. Ib. Cᄃ. Act.Fis. Dep. 
significativo del tiempo de la prueba en el grupo que realizaba estiramientos pasivos $(p<0.05)$.

\section{CONCLUSIONES.}

En función de los resultados obtenidos en las distintas variables de estudio seleccionadas, hemos comprobado que la aplicación de un calentamiento en el que se incluyen ejercicios de estiramiento pasivo no produce mejoras en el rendimiento de la fuerza explosiva y la velocidad.

A pesar de que las diferencias obtenidas al comparar los dos protocolos no han sido en todos los casos significativas, los resultados han mostrado una disminución del rendimiento en todas las variables salvo en el test $\mathrm{CMJ}$, aunque sus beneficios no han sido significativos.

\section{REFERENCIAS BIBLIOGRÁFICAS}

1. Duncan J, Wenger HA, Grenn HJ. Evaluación fisiológica del deportista. Barcelona: Paidotribo; 2000.

2. Fernández $M$, Da Silva $F$. Bases teórico-prácticas del calentamiento de competición en fútbol. Lecturas: Educación Física y Deportes. Revista Digital. 1997;2. Disponible en http://www.efdeportes.com/efd5/mfp51 .htm.

3. Hernández R, Santana A, Suárez L. La preparación inicial de la unidad o clase de entrenamiento. Lecturas: Educación Física y Deportes. Revista Digital. 2001;6. Disponible en http://www.efdeportes.com/efd32/unida d.htm.

4. Rosales A, Rosales J, Batista C, Frómeta C, Leyva S. Algunas consideraciones sobre el calentamiento deportivo. Revista Electrónica Granma Ciencia. 2004; 8. Disponible en http: //www.grciencia.granma.inf.cu/vol8/no. 1/Resumen/2004 0801 r05.htm.

Rev. Ib. CC. Act.Fis. Dep.
5. Bangsbo J. Entrenamiento de la condición física en el fútbol. Barcelona: Paidotribo; 2002.

6. Dadebo B, White J, George K. A survey of flexibility training protocols and hamstring strains in professional football clubs in England. $\mathrm{Br} \mathrm{J}$ Sport Med. 2004;38:388-94.

7. Davis DS, Ashby PE, McCale KL, McQuain JA, Wine JM. The effectiveness of 3 stretching techniques on hamstring flexibility using consistent stretching parameter. J Strength Cond Res. 2005;19:27-32

8. Fernández M, Da Silva F. Modelo aplicativo del calentamiento de competición en fútbol. Lecturas: Educación Física y Deportes. Revista Digital. 1997;2. Disponible en http://www.efdeportes.com/efd7/mfp7. htm.

9. Garcia JM, Navarro M, Ruiz JA. Planificación del entrenamiento deportivo. Madrid: Gymnos; 1996.

10. Leyva C, Castro R. El calentamiento para la actividad físico-deportiva. Lecturas: Educación Física y Deportes. Revista Digital. 2001;7. Disponible en http://www.efdeportes.com/efd36/calen t.htm.

11. Russell $T$ y Bandy WD. Eccentric training and static stretching improve hamstring flexibility of high school males. JAT. 2004;39:254-8.

12. Sánchez DL, Donoso $A$. Fundamentos básicos del calentamiento en el fútbol base II: el calentamiento en el entrenamiento y el calentamiento en el partido. Lecturas: Educación Física y Deportes. Revista Digital. 2003;9. Disponible en http://www.efdeportes.com/efd65/futba se.htm.

13. Decoster LC, Scanlon RL, Horn KD, Cleland J. Standing and supine hamstring stretching are equally effective. JAT. 2004; 39;330-4.

14. Thacker SB, Gilchrist J, Stroup DF, Kimsey CD. The impact of stretching on sports injury risk: a systematic review of the literature. Med Sci Sport Ex. 2004;36:371-8.

15. Burkett LN, Phillips WT, Ziuraitis J. The best warm-up for the vertical jump in college-age athletic men. J Strength Cond Res. 2005;19:673-6. 
16. Cornwell A, Nelson AG, Heise GD, Sidaway B. Acute effects of passive muscle stretching on vertical jump performance. J Hum Mov. 2001;40:307-24.

17. Cornwell A, Nelson AG, Sidaway B. Acute effects of stretching on the neuromechanical properties of the triceps surae muscle complex. Eur $\mathrm{J}$ Appl Physiol. 2002;86:428-34.

18. Fletcher IM, Jones $B$. The effect of different warm up stretch protocols on $20 \mathrm{~m}$-sprint performance in trained Rugby Union Players. J Strength Cond Res. 2004;18:885-8.

19. Kokkonen J, Nelson AG, Cornwell A. Acute muscle stretching inhibits maximal strength performance. Res $Q$ Exerc Sport. 1998;69:411-5.

20. Young WB, Behm DG. Effects of running, static stretching and practice jumps on explosive forcé production and jumping performance. J Sports Med Phys Fitness. 2003;34:119-24.

21. Prentice WE. Técnicas de rehabilitación en la medicina deportiva. Barcelona: Paidotribo; 1997.

22. Nelson AG, Driscoll $N$, Landin D, Young M, Schexnayder I. Acute effects of passive muscle stretching on sprint performance. J Sports Science. 2005;23:449-54.

23. Clanton TO, Coupe KJ. Hamstring strains in atheletes: Diagnosis and treatment. J Am Acad Orthop Surg. 1998:6:237-48.

24. Díaz $C$, Droguett $H$, Enríquez J, Escobar M. Musculatura isquiotibial: referencias anatómicas, sobreestiramiento y flexibilidad. Kinesiología. 2002;67:50-5.

25. Herbert R., Gabriel M. Effects of stretching before and after exercising on muscle soreness and risk of injury: systematic review. BMJ. 2002;325:468-70.

26. Bloomfield J, Wilson G. Flexibility in sport. In: Elliot B \& Mester J, eds. Training in Sport: Applying Sport Science (edited by BC Elliot). Chichester: Wiley; 1998. p.239-85.

27. Shrier I, Gossal K. Myths and Truths of Stretching: Individualized Recommendations for Healthy Muscles. Phys Sportsmed. 2000;28:57-63.

Rev. lb. Cᄃ. Act.Fis. Dep.
28. Zito M, Driver D, Parker C, Bohannon R. Lasting effects of one bout of two 15-second passive stretches on ankle dorsiflexion range of motion. JOSPT. 1997;26:214-21.

29. Arriagada, F.A. y Mendoza, F.J. (2004). Comparación de la efectividad temporal en la técnica de estiramiento estático pasivo aplicada en la musculatura isquiotiobial acortada de futbolistas sub 16 y sub 17 . Tesis de Licenciatura. Universidad de Chile.

30. Diallo O, Dore E, Duche P, Van Praagh E. Effects of plyometric training followed by a reduced training programme on physical performance in prepubescent soccer players. 2001. J Sports Med Phys Fitness;41:342-8.

31. Gissis I, Papadopoulos C, Kalapotharakos VI, Sotiropoulos A, Komsis G, Manolopoulos E. Strength and speed characteristics of elite, subelite and recreational Young soccer players. Res Sports Med. 2006;14: 205-14.

32. Ferrando J, Schneider JL. Relación entre el CMJ y cambios de dirección en deportes colectivos. RICCAFD. 2013;2:30-5.

33. Boone J, Vaeyens R, Steyaert A, Vanden Bossche L, Bourgois J. Physical fitness of elite Belgian soccer players by player position. J Strength Cond Res. 2012;26:2051-7.

34. Di Salvo V, Pigozzi F. Physical training of football players based on their positional roles in the team. J Sports Med Phys Fitness. 1998;38:294-7.

35. Gil SM, Gil J, Ruiz F, Irazusta A, Irazusta J. Physiological and anthropometric characteristics of young soccer players according to their playing position: relevance for the selection process. J Strength Cond Res. 2007;21:438-45.

36. Lago-Peñas C, Casais L, Dellal A, Rey E, Domínguez E. Anthropometric and Physiological characteristics of young soccer players according to their playing positions: relevance for competition success. J Strength Cond Res. 2011;25:3358-67.

37. Le Gall F, Carling C, Williams M, Reilly T. Anthropometric and fitness characteristics of international, professional and amateur male graduate soccer players an elite youth 
academy. J Sci Med Sport. 2010;13:90-5.

38. Balluerka N, Vergara Al. Diseños de investigación experimental en psicología. Madrid: Prentice-Hall; 2002.

39. Bosco C. La valoración de la fuerza con el test de Bosco. Barcelona: Paidotribo; 1994.

40. García JM, Navarro M, Ruiz JA. Pruebas para la valoración de la capacidad motriz en el deporte. Madrid: Gymnos; 1996.

41. Bompa TO. Entrenamiento de la potencia aplicado a los deportes. La pliometría para el desarrollo de la máxima potencia. Barcelona: Inde; 2004.

42. Bosco C. La fuerza muscular. Aspectos metodológicos. Barcelona: Inde; 2000.

Rev. Ib. CE. Act.Fis. Dep. 